\title{
LOS SALARIOS EN URUGUAY, 1930-1950
}

\author{
WAGES IN URUGUAY, 1930-1950
}

\author{
Jorge Notaro* \\ Universidad de la República, Montevideo, Uruguay, <jnotaro@netgate.com.uy>
}

\begin{abstract}
Resumen. Se identifican, se examinan y se comparan las principales estadísticas disponibles sobre salarios desde 1930 hasta 1950. En 1943 una ley creó los consejos con integración tripartita para fijar salarios mínimos por categoría ocupacional y por grupo de actividad económica. Esta nueva fuente no había sido explorada y las resoluciones de los consejos permitieron construir un indicador de los niveles de salarios de obreros y empleados de la actividad privada urbana (sin incluir el servicio doméstico) que muestra que los salarios reales aumentaron 38\% desde 1945 hasta 1950. La información disponible para los años anteriores impide construir un índice, pero permite estimar las tendencias principales, la caída de los salarios reales durante la crisis de 1929-1933, la posterior recuperación parcial y los bajos niveles en 1943.
\end{abstract}

Palabras clave: salarios, negociación colectiva, índices de salarios, metodología.

Abstract. The report identifies, examines and compares the main statistical data which are available to study wages between 1930 and 1950. In 1943 a law created the tripartite Wages Councils with the assignment of fixing minimum wages per occupation in economic activities groups. This new source of information was unexplored yet and those decisions allowed designing an indicator of wage levels of urban workers and employees at the private sector that shows a rise in those real wages of 38\% from 1945 to 1950 . The author analyses the series and concludes that the available information before 1944 is unable to build a wages index but allows identifying the main courses, the real wages fall in the 1929-1933 crises, the partial recover later and the low levels in 1943.

Key words: wages, collective bargaining, wages index, methodology.

Fecha de recepción: enero de 2012. Fecha de aceptación: junio de 2012.

* Agradezco los comentarios de los dictaminadores y también a Lucía Castro, Mariángeles Chevalier, Claudio Fernández, Florencia Gariazzo, Luisa Gastambide, Virginia Motta, Santiago Nuñez, Martín Ortiz, Faustina Ubilla y Magdalena Viera, que con su relevamiento sistemático y riguroso de las resoluciones de los consejos de salarios produjeron una base de datos sin la cual este artículo no hubiera sido posible.

Am. Lat. Hist. Econ., año 20, núm. 2, mayo-agosto, 2013, pp. 96-120 


\section{INTRODUCCIÓN}

L

a Ley de Consejos de Salarios de 1943 creó un escenario de encuentro periódico entre trabajadores y empresarios y con presencia del poder ejecutivo, con el cometido de fijar salarios mínimos por categoría, por grupos de actividad económica del sector privado urbano, excluyendo el servicio doméstico. El objetivo principal de este artículo es utilizar esa fuente de información, inexplorada, para construir un índice de salarios para la segunda mitad de la década de 1940. Como objetivo secundario, se propone evaluar la información disponible y la posibilidad de construir un índice para el periodo de 1930 a 1945.

La revisión exhaustiva de la información sobre salarios desde 1920 permitió concluir que las imprecisiones metodológicas impiden construir un índice de salarios hasta 1945, pero es posible estimar las principales tendencias. Los salarios reales de los trabajadores urbanos cayeron durante la crisis de 1929-1933, la recuperación posterior no alcanzó los niveles previos y su nivel en 1937-1938 era inferior al de 1924-1926. Los salarios reales eran bajos y probablemente descendentes a principios de la década de 1940; la creación de los consejos fue un instrumento para aumentarlos, teniendo en cuenta el punto de vista de los empresarios y el Estado como mediador.

Las resoluciones de los consejos de salarios a partir de 1945 permiten construir un índice con los salarios por categoría que se publican en el Diario Oficial, ${ }^{1}$ catalogarlos según los tipos de ocupación de la Clasificación Internacional Uniforme de Ocupaciones (CIUO-88), deflactarlos con el Índice de Precios al Consumo y analizar su evolución. De acuerdo con el índice construido, los salarios reales mínimos de obreros y empleados urbanos del sector privado aumentaron 38\% desde 1945 hasta 1950. El resultado caracteriza la situación y se constituye en un punto de partida confiable para la búsqueda de explicaciones.

Se presentan los objetivos y la dinámica de los consejos, el contexto histórico del periodo y la controversia sobre su creación. Se fundamenta la validez de sus resoluciones para elaborar un índice de salarios y se exhiben los resultados del índice construido. Se contrastan con los resultados de trabajos anteriores y por último se muestran las conclusiones finales, la bibliografía y el anexo metodológico.

\footnotetext{
${ }^{1}$ Cámara de Representantes, "Informe de la comisión investigadora sobre condiciones de vida, salario y trabajo de la clase obrera”, Diario Oficial, núm. 9801, 17 de abril de 1939.
} 


\section{LOS CONSEJOS DE SALARIOS EN URUGUAY}

\section{El debate sobre la creación}

Las relaciones colectivas de trabajo durante las primeras décadas del siglo Xx funcionaron como pactos gremiales voluntarios y bipartitos ${ }^{2} \mathrm{y}$ la Ley 10449 del 12 de noviembre de 1943 que creó los consejos de salarios estableció un marco para la negociación colectiva tripartita. Cada consejo "hará la clasificación por profesiones y categorías de los trabajadores que integran el grupo respectivo, la que será tomada como base para la fijación de los salarios mínimos" (artículo 9) y "fijarán el salario mínimo aplicable a cada categoría" (artículo 15). Se excluyó a los funcionarios públicos, a los trabajadores rurales y del servicio doméstico.

Se reglamentaron las elecciones de los representantes de los trabajadores y los empresarios (decretos 1119/943 del 19 de noviembre de 1943 y 1880/944 del 30 de junio de 1944), se clasificaron las actividades económicas y las empresas en 20 consejos (decreto 815/944 del 21 de abril de 1944) y en 1944 se resolvieron los primeros laudos.

El primer proyecto de ley de creación de los consejos de salarios tripartitos, que tenía el objetivo de fijar salarios mínimos por categoría, para una actividad económica y un territorio definidos, fue presentado en 1912 por el diputado socialista Emilio Frugoni. Estos lineamientos estuvieron presentes en el proyecto presentado por la Comisión de Legislación Social de la Cámara de Diputados y que se aprobó con modificaciones en 1943; también se mantuvieron en la ley aprobada en 2009 (Ley 18566 del 11 de septiembre de 2009).

Plá Rodríguez identificó como principales opositores al proyecto a los legisladores de la corriente mayoritaria del Partido Nacional, orientada por el doctor Luis Alberto de Herrera. Estos argumentaron que los pequeños comercios no podrían pagar mayores salarios y aumentaría la desocupación, se deteriorarían las ganancias de los empresarios y podría ser un estímulo para la agitación social. También se opusieron las principales gremiales empresariales, como la Cámara de Industrias y de Comercio, la Liga de Defensa Comercial, el Centro de Navegación Transatlántica y el Centro de Almaceneros Minoristas, que "presentaron una nota formulando diversas objeciones". ${ }^{3}$

Finch, por el contrario, consideró que "ciertamente, la noción de que era mejor dejar que el tope salarial lo fijaran las fuerzas del mercado o las

\footnotetext{
${ }^{2}$ Barbagelata, Régimen, 1955.

${ }^{3}$ Plá, Salario, 1956, p. 271.
} 
negociaciones bilaterales entre las dos partes de la industria no tuvo representación en el debate legislativo". ${ }^{4}$

\section{El contexto histórico}

El 31 de marzo de 1933 el presidente electo, doctor Gabriel Terra, dio un golpe de Estado con apoyo de las fracciones de derecha de los partidos Colorado y Nacional, y los sectores agroexportadores, la banca y las empresas extranjeras. ${ }^{5}$

Se convocaron nuevas elecciones en 1938 y las ganó el candidato oficial, general Alfredo Baldomir, con la abstención de fracciones importantes de los partidos mayoritarios (el Nacional y el Colorado), pero con la participación de los partidos menores como el Socialista, el Comunista y la Unión Cívica. Baldomir dio un nuevo golpe de Estado en febrero de 1942, excluyendo del gobierno a los sectores acusados de pronazis, lo que contribuyó a un acercamiento con los partidos opositores y las fracciones que no habían participado en la elección. ${ }^{6}$

Durante el proceso de transición se nombró una comisión parlamentaria con el cometido de estudiar la situación de las familias de los obreros. Los resultados mostraron bajos niveles de salarios y malas condiciones de vida, así como desequilibrios entre el capital y el trabajo. ${ }^{7}$ Se restauró plenamente la democracia en 1942, convocando a elecciones que tuvieron la participación de todos los partidos y fracciones; ganó el Partido Colorado y asumió como presidente Juan José Amézaga, que en el discurso a la Asamblea General en marzo de 1943 señaló que "el contrato de trabajo no puede subsistir como un contrato de adhesión en el que predomina la voluntad de una de las partes".

Para Frega, Maronna y Trochón, ${ }^{8}$ la estrategia de desarrollo requería un cambio en la actitud del poder político hacia los trabajadores para aumentar la demanda interna, consolidar la alianza de las organizaciones políticas y sociales antinazis en el contexto de la segunda guerra mundial y fortalecer la base social del gobierno. Refiriéndose al proyecto de creación de los consejos consideraron que "a partir de los años cuarenta se buscará implementar mecanismos de conciliación entre distintos sectores socia-

${ }^{4}$ Finch, Historia, 1989, p. 13, citado por Bértola, "Salarios”, 2004, pp. 22-23, y por Camou, Regulación, 2000, p. 7.

${ }^{5}$ Porrini, Nueva, 2005, p. 57.

${ }^{6}$ Frega, Maronna y Trochón, Baldomir, 1987, p. 26.

${ }^{7}$ Cámara, Informe, 1939.

${ }^{8}$ Frega, Maronna y Trochón, Baldomir, 1987, p. 29. 
les, tratando de evitar radicalizaciones peligrosas para la paz y el orden social". ${ }^{9}$

En la situación económica se destaca el crecimiento hacia afuera por el impulso de las exportaciones ganaderas hasta la crisis de 1929 y posteriormente por la sustitución de importaciones industriales para el mercado interno hasta mediados de la década de $1950 .{ }^{10}$

Durante la crisis cayó el nivel de actividad y aumentó el desempleo. Según Bertino y Tajam, ${ }^{11}$ desde 1930 hasta 1933 el PBI cayó a 19.4\% y el PBI de la industria manufacturera a 12.6\%. En 1930 ambas variables habían alcanzado un máximo y en 1933 un mínimo desde donde comienza la recuperación con fluctuaciones. Según Millot, Silva y Silva ${ }^{12}$ con cifras de la Oficina Nacional de Trabajo, el desempleo alcanzó a 30000 personas en 1930 y llegó a 40000 en 1932-1933, descendiendo en los años siguientes.

Dos canastas medían el costo de vida de la familia obrera. ${ }^{13}$ De acuerdo con la primera, si los salarios nominales no hubieran cambiado, los salarios reales de los obreros habrían bajado en 1929-1930 para volver al nivel de 1928 en los dos años siguientes. Con la segunda, en la misma hipótesis de salarios nominales estables, los salarios reales descienden hasta 1933-1934 y aumentan en 1935.

Las medidas adoptadas en el periodo muestran la preocupación del gobierno dictatorial por atenuar los impactos de la crisis. El aumento del desempleo motivó un conjunto de medidas entre las que se destacan los comedores populares, las obras públicas, un fondo para "trabajos de socorro" que se repartió entre los gobiernos departamentales y fondos a comisiones distribuidoras de trabajo. ${ }^{14}$

Finalizada la crisis mundial, la estrategia de desarrollo conocida como neobatllista acentuó la promoción de la industria y la agricultura. De 1935 a 1945 el crecimiento industrial "se financió, parcialmente, con relaciones de precios favorables al sector manufacturero en detrimento del agro y de los grupos obreros; también se financió parcialmente con subvenciones y créditos oficiales". Terminada la segunda guerra mundial y superadas las restricciones de abastecimiento, el crecimiento industrial se aceleró, las reservas acumuladas permitieron importar bienes de capital y materias primas, mientras que se protegió el mercado interno con aranceles y se limitó la compra de moneda extranjera. Según Faroppa, "el país entró en franco crecimiento y desarrollo manufacturero. Este fue financiado,

\footnotetext{
${ }^{9}$ Frega, Maronna y Trochón, “Consejos”, 1985.

${ }^{10}$ Faroppa, Desarrollo, 1965, pp. 81-84.

${ }^{11}$ Bertino y Tajam, PBI, 1999.

${ }^{12}$ Millot, Silva y Silva, Desarrollo, 1972, p. 255.

${ }_{13}$ Nahum, Estadisticas, 2009, pp. 307 y 310.

${ }^{14}$ Jacob, Uruguay, 1983, pp. 103-109.
} 
entonces, por el agro y por capas altas de consumo, a través de tipos diferenciales de cambio o subvenciones. Desaparecieron en este periodo, en general, las traslaciones de ingresos desde los grupos obreros hacia las capas empresariales." 15

Las conclusiones de Millot, Silva y Silva ${ }^{16}$ coinciden con esa perspectiva. Estimaron una importante transferencia de ingresos que aumentó la plusvalía de la industria manufacturera a partir de 1938, con un máximo entre 1943 y 1945 y un descenso en los dos años siguientes.

\section{El funcionamiento inicial de los consejos de salarios}

En lo que definió como "un breve balance de los resultados", Plá destacó como consecuencias favorables que: 1. "Ha elevado el nivel de los salarios reales de los trabajadores", de acuerdo con las estimaciones oficiales y como indicador indirecto se puede tener en cuenta la defensa que realizan de los consejos las organizaciones sindicales y los partidos de izquierda. 2. "Ha evitado la comisión de grandes injusticias, eliminando los salarios de miseria", superando los casos graves constatados por la comisión parlamentaria "cuya labor provocó la aprobación de la Ley 10449". 3. "Ha estimulado la organización sindical" y se aprecia un importante desarrollo de las mismas "atribuible, en gran parte a la aplicación de la ley". ${ }^{17}$

Como consecuencias desfavorables señaló que: 1. "Ha operado como un fuerte factor inflacionista", ya que cada aumento de salarios fue sucedido por otro de precios que requería un nuevo aumento de salarios y así sucesivamente. 2. "Ha concentrado la atención en el salario de contratación" disminuyendo el interés en el salario social. 3. "Ha constituido un factor de perturbación social" generando situaciones conflictivas.

A principios de la década de 1940 se estaba formando una clase obrera urbana con gran participación de emigrantes del medio rural hacia Montevideo, así como de Europa hacia Uruguay. ${ }^{18}$ En las primeras elecciones de representantes en los consejos votaron algo más de 42000 trabajadores de un total de casi 97000 habilitados en trece grupos, ${ }^{19}$ contribuyendo a su integración y a menor desigualdad en las relaciones de poder con los patrones.

La convocatoria de los consejos de salarios implicó la institucionalización de la lucha de clases al reconocer la existencia de actores sindicales

${ }^{15}$ Faroppa, Desarrollo, 1965, p. 162.

${ }^{16}$ Millot, Silva y Silva, Desarrollo, 1972, p. 247.

${ }^{17}$ Plá, Salario, 1956, pp. 381-384.

${ }^{18}$ Porrini, Nueva, 2005.

${ }^{19}$ Abdala, Consejos, 1946. 
y empresariales con puntos de vista e intereses contradictorios y convocó a la superación del conflicto con la mediación del Estado, aceptando la relación capital-trabajo y el sistema republicano representativo.

\section{LA EVOLUCIÓN DE LOS SALARIOS}

\section{La construcción de un índice de salarios}

En Uruguay el Índice Medio de Salarios elaborado desde 1968 por el Instituto Nacional de Estadística (INE) "fue establecido por la Ley núm. 13728 del 17 de diciembre de 1968 con el objetivo de estimar la evolución de los ingresos corrientes de los trabajadores permanentes en relación con dependencia de los sectores público y privado formal de todo el país". ${ }^{20}$ Los criterios generales definidos en 1968 se mantienen con algunos ajustes que acompañaron las nuevas modalidades contractuales, así como las modificaciones en la importancia relativa de las actividades y categorías.

No incluye a los trabajadores privados en empresas con menos de diez puestos de trabajo ni de las actividades comprendidas en las secciones A (agropecuaria), B (pesca), C (industria extractiva), O (otras actividades de servicios comunitarias, sociales y personales), $\mathrm{P}$ (hogares privados con servicio doméstico) y $Q$ (organizaciones y órganos extraterritoriales) de la Clasificación Internacional Industrial Uniforme (CIIU, revisión 3). ${ }^{21}$ Tampoco incluye a trabajadores con regímenes de ocupación estacional o remunerada exclusivamente en especie.

Se deducen del salario nominal los aportes a la seguridad social a cargo del trabajador y los impuestos a sus ingresos. Se excluyen los pagos por horas extras y extraordinarios, los descuentos por inasistencia o paros y los aumentos por cambios en las condiciones de trabajo. ${ }^{22}$ En todos estos casos se afecta el precio promedio por unidad de tiempo de los ingresos por trabajo sin que se produzca un cambio en el salario.

Se tienen en cuenta tres tipos de ocupación: profesionales y técnicos, empleados, y obreros, y en cada grupo se eligen algunas categorías; se consideran cuatro estratos de empresas según el número de ocupados y las ponderaciones se calculan de acuerdo con la masa salarial por sector y actividad. Se emplea una fórmula del tipo de Laspeyres, en la que el precio utilizado es la remuneración por hora en cada categoría y en cada empresa u organismo:

\footnotetext{
${ }^{20}$ Instituto, "Índice", 2008, p. 1.

${ }^{21}$ Instituto, "Índice", 2003.

${ }^{22}$ Ibid., pp. 12-13.
} 


$$
I P_{L}=\frac{\Sigma p_{1} q_{0}}{\Sigma p_{0} q_{0}}
$$

$I P$ es el índice de precios, $p_{0}$ los salarios como precio del trabajo y $q_{0}$ las cantidades medidas por las horas trabajadas, ambas variables en el periodo inicial o base señalado por el subíndice 0 . La sumatoria implica considerar un conjunto de trabajadores, cada uno con el salario correspondiente a la categoría en una jornada considerada normal. El valor de $p_{1}$ es el salario correspondiente al año siguiente y la cantidad de trabajo $q_{0}$ se considera constante. Como resultado el índice mide los cambios en los salarios líquidos corrientes por la misma cantidad y calidad de trabajo del año base.

\section{Las resoluciones de los consejos como indicador}

La consideración de que las resoluciones de los consejos permiten construir un buen indicador de la evolución de los salarios se apoya en los siguientes fundamentos:

a) El índice de salarios para la industria manufacturera -elaborado por la Oficina de Planeamiento y Presupuesto para 1945-1960- utilizó como fuente los salarios fijados en los consejos. Davrieux explicó que "los datos correspondientes al periodo anterior, 1945-1960, fueron obtenidos en la Oficina de Planeamiento y Presupuesto, que lo construyó de acuerdo con la metodología establecida por el banco central (cuadro IV-i). Este índice está confeccionado a partir de una categoría ocupacional determinada: la de oficial. En las industrias en que no existía tal cargo se toma el que más se asimila. En su elaboración se tienen en cuenta las publicaciones aparecidas en el Diario Oficial de lo resuelto por los Consejos de Salarios y Convenios Colectivos". ${ }^{23}$ Comprende las agrupaciones 20 a 39 de la Clasificación Internacional Industrial Uniforme (CIIU, revisión 3) con exclusión de la agrupación 32, por ser una actividad estatal. ${ }^{24}$ Desde 1961 el Banco Central del Uruguay continuó la serie y agregó industrias extractivas, de la construcción y el comercio. El índice fue utilizado en trabajos académicos sobre la industria manufacturera. ${ }^{25}$

b) En el momento de aprobación de la ley existían algunos sindicatos y algunos convenios colectivos, pero la cobertura era muy baja. Los consejos de salarios obligaron a los patrones a negociar aumentos de salarios.

\footnotetext{
${ }^{23}$ Davrieux, “Salarios”, 1970, pp. 16-17.

${ }^{24}$ Ibid., p. 16.

${ }^{25}$ Por ejemplo, en Bértola, Industria, 1991, pp. 203-204, y Camou, Regulación, 2000, pp. 6-7.
} 
c) Como indicador indirecto cabe mencionar la resistencia patronal desde los orígenes hasta la fecha. Como se señaló anteriormente, se manifestaron contra el proyecto de ley de creación, dejaron numerosas observaciones en los laudos señalando que los aumentos establecidos llevarían a muchas empresas a la quiebra, pudieron bajar los salarios reales durante los periodos en los que los consejos no funcionaron (1968-1984 y 19922004) y continúan en 2012 reivindicando la negociación voluntaria y por empresa.

d) En la comparación con los índices de salarios, las resoluciones de los consejos tienen ventajas y limitaciones. Los índices ponderan las categorías y las actividades, lo que se transforma en una desventaja cuando la importancia relativa cambia y las ponderaciones permanecen. Las resoluciones de los consejos pueden no cumplirse y algunas empresas pagan salarios menores a los establecidos, sobre todo a los trabajadores no registrados, que el índice de salarios del INE tampoco tiene en cuenta. Por otra parte, se pagan salarios mayores a los mínimos establecidos en situaciones excepcionales: al personal de confianza; en las empresas de mayor productividad para captar a los trabajadores más calificados; como remuneraciones variables cuando se cumplen determinadas condiciones; como pagos no registrados, una práctica habitual en empresas que evaden impuestos y aportes a la seguridad social. Finalmente, si las resoluciones de los consejos de salarios fueran irrelevantes, estos no hubieran funcionado durante tanto tiempo ni hubieran generado polémicas desde el largo proceso de creación hasta la actualidad.

En este artículo se elabora un índice de salarios mínimos de obreros y empleados fijados en los consejos de salarios; es decir, trabajadores urbanos de la actividad privada excluyendo el servicio doméstico y las actividades primarias. No incluye las mismas actividades que el índice del Instituto Nacional de Estadística (A, B, C, P y Q, según la CIIU, revisión 3): las remuneraciones en especie, las extraordinarias ni los descuentos. Se diferencia del índice del INE en que no tiene en cuenta a profesionales y técnicos; se trabaja con un censo de categorías y no con una muestra; se tienen en cuenta los salarios nominales y no los líquidos; el INE pondera las actividades y los tipos de ocupación con la masa salarial y el indicador construido con el número de ocupados. La metodología detallada se presenta en el anexo metodológico. 


\section{Los principales resultados}

El índice construido muestra una tendencia ascendente con fluctuaciones y alcanza el máximo en 1950 con 38\% por encima del nivel de 1945 (véase cuadro 1).

Las categorías definidas en cada consejo de salarios se clasificaron en ocho tipos de ocupación de acuerdo con la Clasificación Internacional Uniforme de Ocupaciones (CIUO-88) utilizada por el INE, ${ }^{26}$ adaptada a los objetivos del trabajo. La delimitación de los tipos de ocupaciones se ilustra con algunos ejemplos de categorías (véase cuadro 1).

En la construcción del índice no se tuvo en cuenta a los tipos de ocupaciones 1 y 2 , para lograr un mejor indicador de los salarios de obreros y empleados.

Para los empleados administrativos (tipo de ocupación 3) se observa una caída del salario real en 1945 por la progresiva incorporación de trabajadores con menores niveles de salarios y sindicatos incipientes. Da un salto en 1946, es estable durante el año siguiente y luego presenta una tendencia ascendente que llega a un aumento de 35\% en 1950.

Para los empleados de comercio y servicios (tipo de ocupación 4) la tendencia ascendente presenta una fluctuación a la baja en el segundo semestre de 1947 y alcanza el máximo en 1950 con $60 \%$ por encima del nivel de 1945.

Los salarios reales de los obreros tienen una evolución similar para los tres niveles de calificación, con un máximo en 1950. Para los calificados (tipo de ocupación 5) el aumento en relación con 1945 fue de 28\%; para los semicalificados (tipo de ocupación 6) fue de 33\% y para los sin calificación (tipo de ocupación 7) de 31\%. Los niveles son consistentes con la calificación, siendo mayores los salarios de los oficiales (tipo de ocupación 5), intermedios para los semicalificados (tipo de ocupación 6) y más bajos para los trabajadores sin calificación (tipo de ocupación 7). El promedio ponderado para los obreros de las tres calificaciones fue de 72 pesos en 1945 y de 94 pesos en 1950, siempre a precios constantes de 1943.

En 1950 el salario promedio ponderado para los obreros equivale a 968 dólares de 2011, para los empleados administrativos a 1205 dólares y para los empleados de comercio y servicios a 1426 dólares (véase anexo metodológico).

\footnotetext{
${ }^{26}$ Disponible en Instituto Nacional de Estadística, Manual Guía para la Codificación de Ocupaciones de Actividades, Clasificación Internacional Uniforme de Ocupaciones (cIUO 88) adaptada a Uruguay (CNUO 95), en <http://www.ine.gub.uy/biblioteca/metodologias/codigosocupacion.pdf $>$. [Consulta: 3 de marzo de 2012.]
} 


\section{CUADRO 1. SALARIOS REALES DE OBREROS Y EMPLEADOS DE LA ACTIVIDAD PRIVADA URBANA, 1945-1950ª}

\begin{tabular}{lccccccc}
\hline & \multicolumn{9}{c}{ Tipo de ocupación } & \multicolumn{3}{c}{ Promedio } \\
Semestres & 3 & 4 & 5 & 6 & 7 & ponderado & Indice \\
& & & & & & & \\
1944-I & 101.2 & 75.5 & 89.8 & 79.9 & 75.4 & 84.2 & 107.8 \\
1945-I & 90.4 & 73.8 & 92.4 & 73.1 & 65.5 & 78.0 & 100.0 \\
1945-II & 80.7 & 97.9 & 87.0 & 73.1 & 65.0 & 79.7 & 102.2 \\
1946-I & 85.4 & 113.9 & 94.7 & 76.0 & 65.3 & 85.7 & 109.8 \\
1946-II & 85.7 & 114.2 & 91.7 & 75.0 & 62.2 & 84.4 & 108.2 \\
1947-I & 85.9 & 112.8 & 87.3 & 71.3 & 59.6 & 82.3 & 105.4 \\
1947-II & 86.4 & 106.3 & 87.3 & 70.3 & 60.3 & 81.0 & 103.9 \\
1948-I & 89.9 & 108.5 & 89.7 & 74.0 & 63.4 & 84.0 & 107.7 \\
1948-II & 91.0 & 110.1 & 91.5 & 78.5 & 66.1 & 86.4 & 110.7 \\
1949-I & 92.1 & 117.3 & 92.9 & 78.9 & 68.5 & 88.9 & 114.0 \\
1949-II & 103.5 & 122.0 & 104.3 & 88.0 & 77.7 & 98.0 & 125.6 \\
1950-I & 112.1 & 137.1 & 113.0 & 96.3 & 83.2 & 107.1 & 137.3 \\
1950-II & 117.0 & 138.5 & 116.9 & 98.3 & 87.0 & 110.4 & 141.4 \\
\hline
\end{tabular}
$1945=100$

${ }^{a}$ Excluyendo al servicio doméstico. En pesos de 1943 e índice base de enero-junio de

Nota: 1. personal directivo y profesionales universitarios -predominantemente negocian sus salarios directamente con los dueños de la empresa-; 2. técnicos y profesionales de nivel medio; 3 . empleados de oficina, incluye a los jefes de sección, auxiliares de todo nivel y cajeros; 4. empleados del comercio y los servicios -vendedores, promotores, corredores, comisionistas, meseros, cocineros-; 5 . oficiales y artesanos, obreros calificados incluyendo capataces, supervisores de planta y choferes; 6 . obreros semicalificados, medios oficiales y otras categorías que hacen referencia a alguna calificación; 7. trabajadores no calificados, peones, serenos, limpiadores y porteros; 8. Aprendices -se denominan así en los laudos y se considera también a las categorías que tienen un límite de edad hasta 21 años.

Fuente: salarios nominales por categoría fijados en los consejos de salarios, deflactados por Índice de Precios al Consumo del INE.

\section{CONTRASTACIÓN CON LA INFORMACIÓN DISPONIBLE}

Se comparan los resultados obtenidos con la información disponible diferenciando los datos anteriores y posteriores a la creación de los consejos. 


\section{El periodo 1914-1943}

El Ministerio de Industrias ${ }^{27}$ publicó información de salarios sin precisiones metodológicas, que deflactada por una canasta de costo de vida de una familia obrera ${ }^{28}$ y promediando algunos años, permitió obtener los resultados que se presentan en el cuadro 2.

Durante la década se aprecia un aumento de los salarios reales de $30.3 \%$ en la industria manufacturera y de $35.9 \%$ en la empresa de tranvías, en un contexto de crecimiento y baja inflación. En el mismo documento se estimó que en 1926 dos tercios de los asalariados de la industria y el comercio, 60400 personas, tenían un ingreso mensual inferior a 50 pesos (64.9 pesos de 1943) que les impedía cubrir el costo de vida de la familia obrera, estimado en 57.50 pesos para ese año. Como se ha visto, con los consejos funcionando para la mayor parte de las actividades, en $1945 \mathrm{el}$ salario promedio de los obreros se estimó en 72 pesos a precios de 1943, 15\% por encima del salario de la industria manufacturera en 1924-1926.

El Ministerio de Industrias y Trabajo ${ }^{29}$ difundió información de salarios de las industrias extractivas, catorce ramas de la industria manufacturera y de la industria de la construcción, que se presenta en el cuadro 3 deflactada. En el informe se explicó que

los salarios promediales se calculan sobre la base del promedio del salario efectivo recibido por los obreros. Es el cociente del total de lo pagado en jornales con respecto al número de obreros. En su monto entran dos factores en juego: el mayor número de horas promedio trabajadas por cada obrero, de acuerdo con la necesidad de la industria y el aumento o disminución del precio promedio de los jornales.

Se aprecia que la caída de los salarios reales que produjo la crisis de 1929-1933 no se recuperó plenamente en los años siguientes, ya que los salarios reales de 1938-1939 son significativamente inferiores a los de 1924-1926.

En la ley aprobada en noviembre de 1943 se eliminó el artículo 16 del proyecto que proponía fijar en 50 pesos mensuales los salarios mínimos para el comercio, la industria y los servicios públicos prestados por privados, lo que permite suponer que era un aumento importante. Si se considera una escala por nivel de calificación similar a la que muestran los consejos, la remuneración de los trabajadores sin calificación sería 10\% menor al promedio, es decir, 43 pesos en la industria manufacturera y

\footnotetext{
${ }^{27}$ Ministerio, Salario, 1927.

${ }^{28}$ Nahum, Estadisticas, 2009, p. 307.

${ }^{29}$ Ministerio, Sintesis, 1940.
} 
CUADRO 2. SALARIOS REALES A PRECIOS DE 1943

\begin{tabular}{lcc}
\hline Periodos & Tranvías & $\begin{array}{c}\text { Industria } \\
\text { manufacturera }\end{array}$ \\
1914-1917 & 50.1 & 47.8 \\
$1918-1920$ & 47.7 & 45.8 \\
$1921-1923$ & 60.1 & 54.2 \\
$1924-1926$ & 68.1 & 62.3 \\
\hline
\end{tabular}

Fuentes: salarios del Ministerio de Industria, 1927, y deflactor de Nahum, 2009.

CUADRO 3. SALARIOS REALES PROMEDIO

DE LA INDUSTRIA A PRECIOS DE 1943

\begin{tabular}{cccc}
\hline Años & Extractivas & Manufactureras & Construcción \\
& & & \\
1938 & 52.5 & 50.7 & 45.8 \\
1939 & 48.4 & 47.8 & 43.5 \\
\hline
\end{tabular}

Fuentes: salarios del Ministerio de Industrias y Trabajo, 1940, y deflactor de Nahum, 2009.

39 en la construcción, por lo que un mínimo de 50 pesos significaba un aumento de $16 \%$ en la manufactura y de $28 \%$ en la construcción. Entre 1939 y 1943 el aumento del Índice de Precios al Consumo del InE fue de 19\%, lo que fortaleció la necesidad de encontrar formas de negociación de aumentos de salarios. ${ }^{30}$

Según Millot, Silva y Silva ${ }^{31}$ el salario mensual de los obreros a precios de 1943 era de 45.2 pesos en $1938-1939$ y de 47.44 pesos para 1943. El primero se sitúa $8 \%$ por debajo del promedio que proporciona el Ministerio de Industria, lo que implica que para estos autores la recuperación de los impactos de las crisis de 1929 sobre los salarios fue menor que para el Ministerio de Industria. El segundo es igual al del Ministerio de Industria para 1938-1939, lo que implica 8\% de aumento del salario real de la industria manufacturera en el periodo previo a la instalación de los consejos, lo que parece poco probable teniendo en cuenta la baja cobertura de la

\footnotetext{
${ }^{30}$ Nahum, Estadísticas, 2009, p. 307.

${ }^{31}$ Millot, Silva y Silva, Desarrollo, 1972, pp. 209 y 231.
} 
negociación colectiva y el importante aumento del Índice de Precios al Consumo en esos años.

Durante 1944, mientras se procesaba la instalación de los consejos, el Parlamento aprobó varias leyes extendiendo progresivamente un aumento de salarios a todas las actividades privadas, excluyendo a los trabajadores rurales y del servicio doméstico. ${ }^{32} \mathrm{El}$ aumento fue de $10 \%$ en todos los casos y en distintos momentos, lo que permite estimar un aumento promedio anual en torno a $6 \%$ en los salarios nominales de la industria, similar al $5.2 \%$ registrado por Millot, Silva y Silva.

$\mathrm{El}$ análisis realizado permite suponer que los salarios eran bajos y probablemente descendentes en términos reales a principios de la década de 1940. La creación de los consejos de salarios se aprobó como un instrumento para aumentar los haberes, teniendo en cuenta el punto de vista de los empresarios y con el Estado como mediador. El poder ejecutivo se reservó la facultad de modificar las resoluciones de los consejos si consideraba que los salarios fijados eran muy bajos (artículo 15).

\section{El periodo 1944-1950}

Se pueden comparar los resultados obtenidos con los de un documento del Ministerio de Industria y Trabajo ${ }^{33}$ con información sobre el salario nominal para Montevideo en algunas actividades de la industria para tres categorías: peón, medio oficial y oficial o sus equivalentes (véase cuadro 4). El Ministerio de Industria y Trabajo calculó los salarios explicitando que "para facilitar y representar mejor el ritmo de la espiral aumentativa del salario, de cada una de las actividades, y por año, se extrajo el jornal promedio mediante la siguiente fórmula: (jornal $\mathrm{A}+$ jornal $\mathrm{B}+$ jornal C)/3. Con el jornal promedio obtenido, se estableció el aumento en moneda y porcentual de cada actividad entre 1943-1950."

Los salarios de los laudos son menores a los estimados por el Ministerio de Industrias y Trabajo en los tres niveles. Se puede explicar por dos razones: el índice construido incluye todas las actividades, no solamente la industria manufacturera, y para todo el país, no sólo Montevideo. En ambos casos se incorporan actividades con menores niveles de salarios.

Se observan también varias coincidencias entre ambas estimaciones: a) los niveles de salarios son consistentes con las calificaciones de los trabajadores; b) para las tres calificaciones la tendencia es ascendente en el

\footnotetext{
${ }^{32}$ Plá, Salario, 1956, t. I, pp. 428-473.

${ }^{33}$ Ministerio, Clasificación, 1953, pp. 775-806.
} 


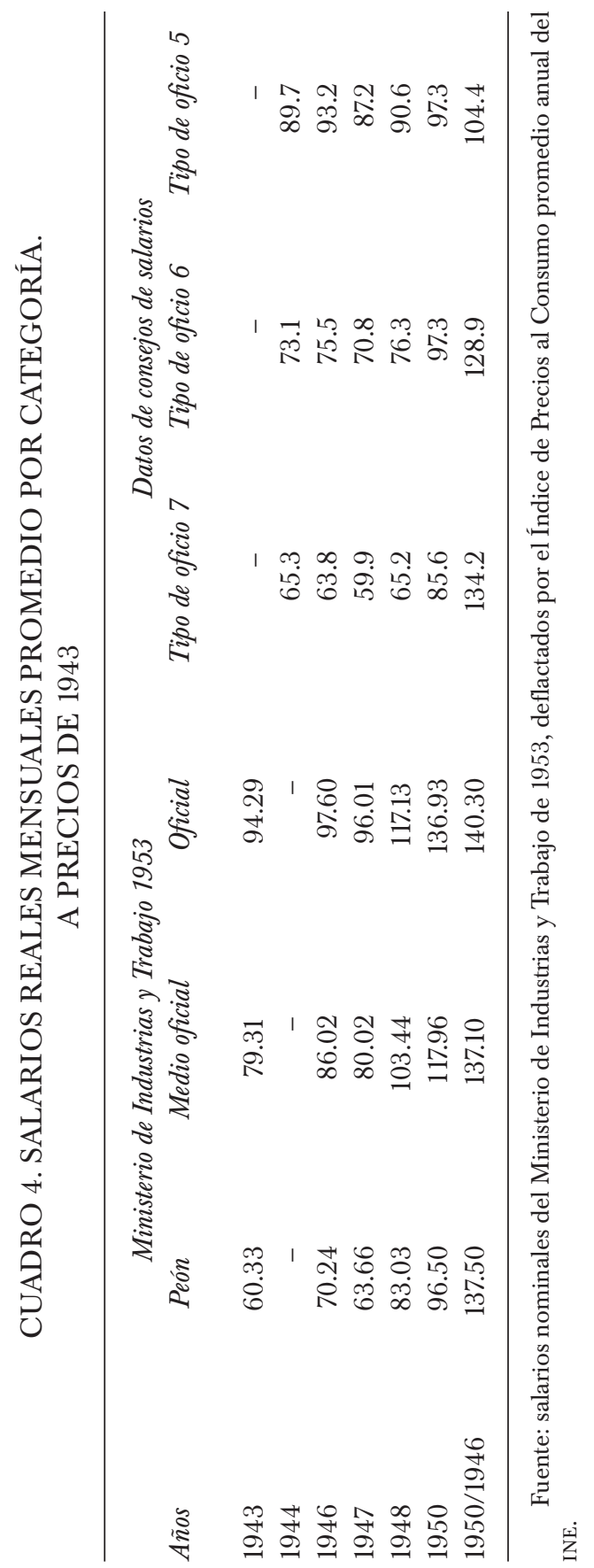


periodo; c) en 1947 ambos indicadores presentan una fluctuación a la baja. Se concluye que los resultados de los dos indicadores son compatibles.

Los índices construidos con posterioridad al funcionamiento de los consejos se evalúan con dos criterios. En primer lugar el grado de cumplimiento de los requisitos para la construcción de índices de salarios vistos en "la construcción de un índice de salarios", lo que permite analizar la transparencia metodológica entendida como la posibilidad de que el lector disponga de los elementos para recorrer el camino del investigador y concluir sobre la calidad de la información. En segundo lugar, comparando los resultados con otra información confiable e interpretándolos en su contexto. No se incluye a Davrieux ${ }^{34}$ ni al Instituto de Economía ${ }^{35}$ que utilizaron el índice elaborado por la Oficina de Planeamiento y Presupuesto (OPP) del Banco Central del Uruguay. Los resultados se presentan sintéticamente en el cuadro 5 y luego se analizan con detalle.

El Índice Medio de Salarios del INE define las actividades comprendidas, la cobertura geográfica, el sector institucional, las empresas encuestadas, el tipo de contrato, los tipos de ocupación, las categorías, los tipos de ingresos, los criterios de ponderación y el deflactor.

Millot, Silva y Silva ${ }^{36}$ presentan el salario mensual promedio de los obreros y el sueldo mensual promedio de los empleados a precios corrientes para trece ramas de la industria manufacturera y calculan un "salario nominal promedio de los obreros" que deflactan por el costo de vida de una familia obrera. No explicitan la cobertura geográfica, el sector institucional ni los criterios de ponderación. Los resultados generan algunas interrogantes: a) en 1930 y 1936 los salarios son similares, lo que implica que en este último año se habían recuperado del impacto de la crisis; b) los salarios reales descienden hasta 1940 y aumentan 14\% entre este año y 1943, lo que no corresponde con las preocupaciones que dieron origen a la creación de los consejos; $c$ ) en 1947 se presenta un aumento del salario real de $2.3 \%$, poco probable en un año con un aumento de $15 \%$ del Índice de Precios al Consumo.

El índice OPP-Banco Central del Uruguay para la industria manufacturera tiene casi todas las precisiones requeridas y considera el salario de una categoría de oficial para cada agrupación industrial, de modo que no incluye a los obreros sin calificación ni a los empleados. El Instituto de Economía $^{37}$ difunde el índice para el periodo de 1961-1969 explicando que incluye "los salarios nominales laudados para la actividad privada en

\footnotetext{
${ }^{34}$ Davrieux, "Salarios", 1970.

${ }^{35}$ Instituto, Estadísticas, 1969.

${ }^{36}$ Millot, Silva y Silva, Desarrollo, 1972, pp. 231- 235.

${ }^{37}$ Instituto, Estadísticas, 1969, pp. 92 y 111.
} 
CUADRO 5. CARACTERÍSTICAS DE LOS ÍNDICES DE SALARIOS

\begin{tabular}{lcccccccc}
\hline Índices & $A$ & $B$ & $C$ & $D$ & $E$ & $F$ & $G$ & $H$ \\
Índice Medio de Salarios-INE, 1968 & 1 & 1 & 2 & 2 & 2 & 2 & 1 & 1 \\
OPP-Banco Central del Uruguay, 1945 & 2 & 2 & 2 & 2 & 2 & 1 & 1 & 1 \\
Millot et al., 1972 & s. d. & s. d. & 1 & 1 & 1 & 1 & 2 & 2 \\
Trajtenberg, 1973 & 2 & 2 & 2 & 2 & 2 & 1 & 1 & 1 \\
Bértola et al., 1999 & s. d. & 4 & s. d. & s. d. & s. d. & 1 & 2 & 2 \\
Notaro, 2011 & 1 & 2 & 1 & 2 & 1 & 1 & 1 & 1 \\
\hline
\end{tabular}

Nota: A. Cobertura geográfica: 1. nacional, 2. Montevideo. B. Sector institucional: 1. todos, 2. privado, 3. público, 4. cambiante. C. Contratos: 1. todos, 2. permanentes. D. Tipos de ocupación: 1. todos, 2. parte. E. Categorías: 1. todas, 2. parte. F. Salarios: 1. nominales, 2. líquidos. G. Ponderaciones: 1. explícitas, 2. no se explicitan. H. Deflactor: 1. Índice de Precios al Consumo del INE, 2. otros.

el departamento de Montevideo. Se eligieron las categorías de salarios más representativas en cada grupo de actividad y se ponderaron los distintos grupos de acuerdo con las cifras proporcionadas por el Registro Industrial de 1957."

Trajtenberg, con la colaboración de Davrieux, ${ }^{38}$ presentó un índice de salarios reales del sector privado de Montevideo citando como fuentes a la OPP de 1945 a 1961 y al Banco Central del Uruguay desde 1961 hasta 1967. Señaló que "las cifras anteriores a 1949 no son excesivamente confiables, pero no cabe duda de que 1950 señala ya un nivel considerable para lo que serán los salarios a partir de ese momento".

Bértola y otros ${ }^{39}$ construyeron un índice general desde 1907 hasta 1926 tomando el jornal de un trabajador no calificado de la construcción del sector público; desde 1926 hasta 1936 citaron como fuente los anuarios estadísticos y desde este último año hasta 1968 a la Dirección General de Asuntos Económicos del Ministerio de Industrias y a la publicación Estudios y Coyuntura del Instituto de Economía. ${ }^{40}$ Se empalmaron los resultados de diferentes actividades económicas y categorías laborales (construcción privada para el sector público e industria manufacturera). No se explicitó la cobertura geográfica, los tipos de ocupación ni los criterios de ponderación. No se aclaró el tratamiento de las clases de actividad A, B, C, P

${ }^{38}$ Trajtenberg, "Evolución”, 1973.

${ }^{39}$ Bértola et al., Southern, 1999.

${ }^{40}$ La referencia correcta es Trajtenberg, "Evolución”, 1973. Para el sector privado desde 1948 a 1972 y para el sector público desde 1961 hasta 1972. 
y Q así como de las empresas públicas como Administración Nacional de Combustibles, Alcohol y Portland, creada en 1931 con actividad en A (industrias extractivas) y B (agropecuaria), así como en las agrupaciones 21 (bebida) y 32 (combustibles) de la industria manufacturera.

Los resultados son discutibles, teniendo en cuenta que: a) los salarios nominales aumentan 17\% desde 1928 hasta 1932 y descienden 7\% desde este último año hasta 1935; es poco probable el aumento durante la crisis, que como se ha visto implicó la caída del nivel de actividad y un importante aumento del desempleo; b) en 1944 aumentan 9.4\%, más de 50\% que los aumentos legales; $c$ ) los salarios reales se contraen 10\% en 1945 con el pleno funcionamiento de los consejos; d) los salarios reales aumentan 20\% en 1947 con un aumento de 15\% del Índice de Precios al Consumo en el mismo año. En síntesis, de 1945 a 1950 las fluctuaciones son demasiado grandes en un contexto económico, social y político sin cambios significativos y contradicen las tres fuentes disponibles para el periodo.

La serie tuvo un efecto dominó, ya que se utilizó en varios trabajos. ${ }^{41}$ En el Banco de Datos de la Unidad Multidisciplinaria de la Facultad de Ciencias Sociales ${ }^{42}$ este cuadro aparece con el número 2 y se repite como número 5 como salario de la industria manufacturera, con un valor distinto en 1920, el aumento durante la crisis de 1929-1933 y las fluctuaciones entre 1945-1950. En el cuadro 3, el banco presenta un índice de salarios de la industria manufacturera citando como fuentes a Millot, Silva y Silva y a Davrieux, que como se ha señalado tienen diferentes metodologías. Las principales interrogantes se plantean en: $a$ ) el aumento de $11.5 \%$ desde 1940 a 1943 ; b) la estabilidad en 1944, año de aumentos obligatorios por ley y que el índice de Millot, Silva y Silva registró; c) el aumento en 1947, así como las caídas en 1945 y 1949.

Camou señaló que "durante la década de 1930, las condiciones del mercado de trabajo empeoran para los asalariados". ${ }^{43}$ Por la crisis, el aumento de la desocupación y la regulación de los salarios básicamente por la competencia. Sin embargo, en el cuadro 2 del trabajo de Camou, ${ }^{44}$ los salarios reales de la industria manufacturera aumentan en 1929, permanecen estables hasta 1931, bajan 5\% en 1933 y 1934, y desde este año inician una tendencia ascendente. En la planta La Industrial de la empresa Campomar aumentan 22.6\% hasta 1932, en el periodo de 1942-1949 el aumen-

${ }^{41}$ Bértola, Ensayos, 2000, pp. 91-119, y "Salarios", 2004, pp. 23-30; Camou, Regulación, 2000, pp. 5-6, "Industrialización", 2001, Cambios, 2011, y Camou y Maubrigades, "Desafío", 2006; Arnábal, Bertino y Fleitas, Revisión, 2010, y Lara, Evolución, 2010.

${ }^{42}$ Disponible en $<$ http://www.fcs.edu.uy/pagina.php?PagId=801\&CatId=111\&SubCatId=243>.

${ }^{43}$ Camou, Regulación, 2000, p. 1.

${ }^{44}$ Ibid., p. 15. 
to se estima en $93 \%,{ }^{45}$ y en $97.3 \% .{ }^{46}$ Los resultados no se contrastan con los salarios fijados en los laudos en el subgrupo de Campomar. Camou ${ }^{47}$ presentó posteriormente una gráfica de los salarios reales de la industria citando la misma fuente pero con una evolución diferente, aumento desde 1929 a 1935, descenso hasta 1940, aumento al año siguiente y descenso hasta 1946.

Bértola $^{48}$ señaló que "el caso uruguayo muestra un deterioro similar al argentino y brasileño en la década de 1930, aunque una mejora relativa en la posguerra", afirmación que contradice la serie de salarios citada..$^{49} \mathrm{La}$ conclusión "la protección permitía cubrir a las industrias por los costos adicionales del salario" es un fenómeno que forma parte de las transferencias de ingresos que investigaron Faroppa ${ }^{50}$ y el Instituto de Economía ${ }^{51}$ que no fueron citados. En el análisis del papel de los consejos de salarios en un trabajo posterior, Bértola ${ }^{52}$ afirmó que "sin lugar a dudas, los consejos de salarios contribuyeron al proceso mediante el cual los salarios crecieron rápidamente hasta 1950 y a niveles históricos nunca retomados, pero sería un grueso error atribuir a los propios consejos esos logros". Explicó la evolución de los salarios con un enfoque original, que no forma parte de las teorías conocidas, ${ }^{53}$ por tres aspectos: los términos de intercambio, el control del comercio exterior y el aumento de la productividad.

Lara explicó la metodología del Índice de Salarios de la industria manufacturera del Banco de Datos de la Unidad Multidisciplinaria de la Facultad de Ciencias Sociales para el periodo de 1930-1969 que utiliza las estimaciones de Millot, Silva y Silva, Davrieux y Bértola y otros. Consideró que por el efecto del empalme de los dos primeros índices, tomando el valor del segundo para el año común de 1948 y ajustando hacia atrás, la serie "representa la evolución de los salarios de la categoría oficial"; la serie de Millot, Silva y Silva incluye a todas las categorías y su cobertura no se modifica por un cambio de base. ${ }^{54}$ Los índices de salarios nominales presentados en el anexo estadístico de su trabajo son el resultado de empalmar series con distintos sectores institucionales, actividades, tipos de ocupación, categorías, tipos de contratos y tipos de ingresos. La cobertura geográfica y los criterios de ponderación no se explicitan.

${ }^{45}$ Ibid., p. 7.

${ }^{46}$ Ibid., p. 12

${ }^{47}$ Camou, Cambios, 2011, p. 13.

${ }^{48}$ Bértola, Ensayos, 2000, pp. 113-114.

${ }^{49}$ Bértola, Southern, 1999.

${ }^{50}$ Faroppa, Desarrollo, 1965, pp. 161-162.

${ }^{51}$ Instituto, Proceso, 1969.

${ }_{52}$ Bértola, "Salarios", 2004, pp. 23-30.

${ }^{53}$ Amarante y Bucheli, Negociación, 2007, pp. 5-24.

${ }^{54}$ Lara, Evolución, 2010, p. 4. 


\section{CONCLUSIONES FinAleS}

Las resoluciones de los consejos fijando salarios mínimos por categoría y por grupo de actividad desde 1944 permiten construir un índice de salarios de obreros y empleados de la actividad privada urbana con exclusión del servicio doméstico. En su elaboración se explicitaron las actividades comprendidas, la cobertura geográfica, el sector institucional, el tipo de contrato, los tipos de ocupación, las categorías, los ingresos incluidos, los criterios de ponderación y el deflactor. El lector dispone de los elementos para recorrer el camino del investigador y concluir sobre la calidad de la información y los fundamentos de las conclusiones.

De acuerdo con este índice, los salarios reales mínimos de obreros y empleados aumentaron desde 1945 hasta 1950 en 38\%, con una fluctuación a la baja en 1947, año en el que se registró alta inflación. El indicador construido caracteriza la situación y se constituye en un punto de partida confiable para la búsqueda de explicaciones.

La revisión exhaustiva de la información sobre salarios disponible desde 1920 permitió concluir que las imprecisiones metodológicas impiden construir un índice hasta 1945, pero permiten estimar las tendencias principales. Los salarios de la mayor parte de los trabajadores urbanos se ubicaban por debajo del costo de vida de la familia obrera a mediados de la década de 1920; cayeron durante la crisis de 1929-1933; la recuperación posterior no alcanzó los niveles previos, y los salarios reales en 1937-1938 eran inferiores a los de 1924-1926.

La encuesta de la comisión parlamentaria de 1939 mostró que los salarios eran muy bajos y las condiciones de vida de las familias obreras muy malas. Entre 1939 y 1943 el aumento del Índice de Precios al Consumo del INE fue de 19\%, lo que fortaleció la necesidad de encontrar procedimientos para aumentar los salarios.

En el momento de aprobación de la ley (noviembre de 1943) existían algunos sindicatos y convenios colectivos con una cobertura muy baja. La negociación de salarios requería la aprobación de los patrones y tenía lugar en condiciones de desigualdad en las relaciones de poder. El Parlamento aprobó varias leyes durante 1944, aumentando 10\% los salarios para todas las actividades incluidas en los consejos.

El análisis realizado permite suponer que los salarios eran bajos y probablemente descendentes en términos reales a principios de la década de 1940. La creación de los consejos se aprobó como un instrumento para aumentar los salarios, teniendo en cuenta el punto de vista de los empresarios y con el Estado como mediador. El poder ejecutivo se reservó la facultad de modificar las resoluciones de los consejos si consideraba que los salarios fijados eran muy bajos. 
La convocatoria obligó a negociar; la elección de representantes de los trabajadores estimuló el desarrollo de las organizaciones sindicales y estas promovieron el aumento de salarios. Los patrones los transformaron en máximos y los sindicatos con el apoyo de la Inspección Nacional del Trabajo y Servicios Anexados aseguraron su cumplimiento en la mayor parte de los casos.

\section{ANEXo METODOLÓGICO}

El principal antecedente son cinco monografías de grado que abordaron el análisis de los 20 grupos de consejos de salarios creados en 1943, que construyeron una base de datos con las fotografías de los laudos y formularios con los salarios por categoría. Fue el punto de partida homogeneizando la información y diseñando los procedimientos de elaboración del índice que se presenta en el artículo.

$\mathrm{El}$ acceso a los laudos del periodo investigado requiere un trámite en la Biblioteca Nacional, pero se pueden ver los vigentes, que continúan fijando salarios mínimos por categoría, en la página del Ministerio de Trabajo y Seguridad Social. ${ }^{55}$ Se clasificaron las categorías por tipos de ocupación y se hizo un promedio aritmético de los salarios nominales mínimos por categoría, se deflactaron por el Índice de Precios al Consumo de la Dirección General de Estadística y Censos (actualmente INE) con base en 1943 , se promediaron por semestre y se obtuvieron los salarios reales semestrales promedio por tipos de ocupación.

Para calcular el índice general no se tuvieron en cuenta los tipos de ocupación 1 y 2 , de modo que el indicador representa los salarios de obreros y empleados.

Como la ley se aprobó en noviembre de 1943 se tomó ese año como base del deflactor, lo que permite apreciar más claramente los cambios posteriores de los salarios reales. El Îndice de Precios al Consumo del INE desde 1938 se puede consultar en el sitio web de ese instituto. ${ }^{56}$

La ponderación de los tipos de ocupación en cada consejo de salarios, así como de cada consejo de salarios en el total, se realizó predominantemente con la información del trabajo monográfico de Estela Méndez que procesó la información de los censos y de la encuesta continua de hogares. ${ }^{57}$ También se tuvo en cuenta el número de habilitados para votar

${ }^{55}$ Consúltese $<$ http://www.mtss.gub.uy/index.php?option=com_content\&view=article\&id= $1665 \&$ Itemid $=340>$.

${ }^{56}$ Consúltese $<$ http://www.ine.gub.uy/preciosysalarios/ipc2008.asp?Indicador=ipc >.

${ }^{57}$ Méndez, “Ocupación”, 1983. 
en cada consejo $;^{58}$ el progresivo pasaje de actividades del sector privado al público como los tranvías (1947), el ferrocarril y el agua corriente (1948) y la información de Millot, Silva y Silva por rama de la industria manufacturera sobre el número de obreros y de empleados desde 1938 a $1947^{59}$

Los valores calculados se pueden llevar a precios de 2011 para dar una idea aproximada de lo que representarían actualmente. Hay que tener en cuenta dos cambios en la unidad monetaria de Uruguay: a) por la Ley 14316 del 19 de junio de 1975, 1000 pesos pasaron a ser un nuevo peso; $b$ ) por la Ley 16226 del 29 de octubre de 1991, 1000 nuevos pesos pasaron a ser un peso uruguayo. Por lo tanto, los pesos del periodo analizado se dividen entre un millón y el resultado se multiplica por el valor del Índice de Precios al Consumo de 2011 con base en 1943; el coeficiente que resulta de este procedimiento para estimar los salarios a precios de 1943 en valores de 2011 es 205 987. Por ejemplo, un salario de 68.8 pesos a precio de 1943 equivale a 14172 pesos de 2011 y si se tiene en cuenta que la cotización promedio del dólar en 2011 fue de 20 pesos, equivale a 709 dólares.

\section{Fuentes CONSULTADAS}

\section{Archivos}

Banco de Datos de Economía e Historia Económica, Universidad de la República, Montevideo, 2011, en <http://www.fcs.edu.uy/pagina.php?PagId=801>. [Consulta: 6 de enero de 2012.]

\section{Hemerografia}

Diario Oficial de la República Oriental del Uruguay, Montevideo, Uruguay.

Revista de la Dirección de Asuntos Económicos del Ministerio de Industrias y Trabajo, núm. 1, 1946.

\section{Bibliografía}

Abdala, Salvador, Consejos de salarios. Jurisprudencia, leyes, decretos, laudos, resoluciones, apelaciones, comentarios, Montevideo, Monteverde y Cía., 1946.

${ }^{58}$ Abdala, Consejos, 1946.

${ }^{59}$ Millot, Silva y Silva, Desarrollo, 1972, pp. 219-233. 
Amarante, Verónica y Marisa Bucheli, Negociación salarial colectiva: revisión de la literatura y de la experiencia en Uruguay, 2005-2006, Montevideo, Comisión Económica para América Latina y el Caribe, 2007 (Serie Estudios y Perspectivas, 12).

Arnábal, Rodrigo, Magdalena Bertino y Sebastián Fleitas, "Una revisión del desempeño de la industria uruguaya en el periodo de sustitución de importaciones", ponencia presentada en Séptimas Jornadas de Investigación de Historia Económica, Asociación Uruguaya de Historia Económica, Uruguay, 5 y 6 de agosto de 2010.

Barbagelata, Héctor Hugo, Régimen de los convenios colectivos, Montevideo, Federación Uruguaya de Empleados de Comercio e Industria, 1955, vol. 2 (Colección de Cultura Sindical).

Bertino, Magdalena y Héctor Tajam, El PBI de Uruguay, 1900-1955, Montevideo, Universidad de la República/Comisión Sectorial de Investigación Científica, 1999.

BÉRTOLA, LUIS, La industria manufacturera uruguaya. Un enfoque sectorial de su crecimiento, fluctuaciones y crisis, Montevideo, Centro Interdisciplinario de Estudios sobre el Desarrollo, 1991.

Ensayos de historia económica. Uruguay y la región en la economía mundial 18701990, Montevideo, Ediciones Trilce, 2000.

"Salarios reales y distribución del ingreso, 1930-1968. Contextualizando los consejos de salarios” en AMÉRICO PlÁ RodRÍGuez et al., Los consejos de salarios. Una mirada actual, Montevideo, Fundación de Cultura Universitaria, 2004. Luis Calicchio, María Camou y Gabriel Porcile, Southern Cone Real Wages Compared: A Purchasing Power Parity Approach to Convergent and Divergent Trends, 1870-1996, Montevideo, Universidad de la República, 1999 (Documento de Trabajo, núm. 44).

CAMOU, MARÍA, "La regulación salarial. Estudio de una empresa textil: Campomar y Soulas (1920-1949), Porto Alegre”, ponencia presentada en Primeras Jornadas de Historia Regional Comparada, Fundación de Economía y Estadística, 2000.

"Industrialización y trabajo: un enfoque de la relación salarial desde una empresa textil, 1922-1949”, tesis de maestría, Uruguay, Universidad de la República, 2001.

, "Cambios en la estructura salarial en dos ramas de la industria uruguaya, 1900-1960", trabajo presentado en Quintas Jornadas de Historia Económica, Asociación Uruguaya de Historia Económica/Universidad de la República, 2011. y Silvana Maubrigades, "El desafío de la productividad en la industria 'tradicional' uruguaya" en MARÍA CAMOU y RODOLFO PORRINI, Trabajo e historia en el Uruguay. Investigaciones recientes, Montevideo, Universidad de la República/ Comisión Sectorial de Investigación Científica, 2006.

DAVRIEux, Hugo, "Salarios y acción sindical en el Uruguay. Industria manufacturera, construcción y gobierno central”, tesis de grado, Universidad de la República, Uruguay, 1970. 
FAROPPA, LUIS, El desarrollo económico del Uruguay: tentativa de explicación, Montevideo, Centro de Estudiantes de Ciencias Económicas y de Administración, 1965.

Finch, Henry, Historia económica del Uruguay contemporáneo, 1870-1970, Montevideo, Ediciones de la Banda Oriental, 1989.

Frega, Ana, Mónica Maronna e Ivette Trochón, "Los consejos de salarios como experiencia de concertación”, Cuadernos del Centro Latinoamericano de Economía Humana, vol. 10, núm. 33, 1985, pp. 27-36.

Baldomir y la restauración democrática (1938-1946), Montevideo, Ediciones de la Banda Oriental, 1987 (Temas del Siglo XX, vol. 39).

Instituto de Economía, Facultad de Ciencias Económicas y de AdministraCión, Universidad de la República, El proceso económico del Uruguay, Montevideo, Fundación de Cultura Universitaria, 1969. Estadísticas básicas, Montevideo, Universidad de la República, 1969.

Instituto Nacional de Estadística, "Índice medio de salarios. Ley No. 13728 del 17 de diciembre de 1968 y Ley No. 17649 del 3 de junio de 2003, Metodología. Base diciembre 2002 = 100", Montevideo, 2003, en <http://www.ine.gub.uy/biblioteca/metodologias/Metodologia\%20IMS\%20Diciembre\%202002=100.pdf $>$. [Consulta: 10 de junio de 2011.]

"Índice medio de salarios e índice medio de salarios nominales. Cambio de base, julio de 2008. Nota metodológica", Montevideo, 2008, en <http://www. ine.gub.uy/comunicados/ims/IMS\%20-\%20nota $\% 20$ metodologica.pdf>. [Consulta: 10 de junio de 2011.]

JaCob, Raúl, El Uruguay de Terra, 1931-1938. Una crónica del terrismo, Montevideo, Ediciones de la Banda Oriental, 1983 (Temas del Siglo XX, vol. 11).

LARA, MARÍA CECILIA, Evolución de los salarios nominales y reales para la industria manufacturera y ciertas clases de actividad (1930-1969), Montevideo, Universidad de la República, 2010, en <http://www.fcs.edu.uy/archivos/Salarios\%20sector\%20privado\%20por\%20clases\%20activ_1930_\%201969.pdf $>$. [Consulta: 10 de junio, 2011.]

Méndez, Estela, “Ocupación en Uruguay, 1963-1981”, tesis de licenciatura en Economía, Montevideo, Universidad de la República, 1983.

Millot, Julio, Carlos Silva y Lindor Silva, El desarrollo industrial del Uruguay de la crisis de 1929 a la segunda posguerra, Montevideo, Universidad de la República, 1972.

Ministerio DE Industrias, El salario real (1914-1926), Montevideo, Imprenta Nacional, 1927.

Ministerio de Industrias y Trabajo, Sintesis de la evolución industrial en los años 19381939, Montevideo, Ministerio de Industrias y Trabajo, 1940.

Clasificación industrial de ramas de actividad económica del Uruguay. Digesto de la actuación de los consejos de salarios entre los años 1943-1952. Coste de vida, estudio de precios y salarios a través de los años 1943-1952, Montevideo, Imprenta Nacional, 1953.

Nahum, Benjamín (coord.), Estadísticas históricas del Uruguay, 1900-1950, t. III, Economía, Montevideo, Universidad de la República, 2009. 
Plá Rodríguez, Américo, El salario en el Uruguay: su régimen jurídico, Montevideo, Universidad de la República, 1956, libro segundo, sec. III, núm. LXXXIII.

Porrini, Rodolfo, La nueva clase trabajadora uruguaya (1940-1950), Montevideo, Universidad de la República, 2005.

Trajtenberg, Raúl, "I. La evolución de los salarios hasta 1968”, Estudios y Coyuntura, Instituto de Economía, núm. 3, 1973, Montevideo, pp. 195-217. 